\title{
Role of Lifestyle on Healthy Ageing
}

\author{
Dr. Savneet
}

\begin{abstract}
Life expectancy is increasing around the world, and this is likely to have profound effects on many aspects of society, particularly if these extra years are to be associated with ill health. Lifestyle plays most important role on healthy ageing. An active and controlled lifestyle plays $99 \%$ role in healthy ageing. The study focuses on effect of lifestyle on healthy ageing.

The present study was conducted on a sample of $(N=20)$ Age above 60 years. The instrument used was a questionnaire comprising questions such as Life achievements, Source of inspiration and Role of life style in healthy ageing. An anxiety test (self-designed) was also given to assess the anxiety levels of these subjects.

It was found that people, who followed simple life, ate simple food and exercises had fewer problems and were generally satisfied with life and had healthy social wellbeing.
\end{abstract}

\section{Introduction}

Unveiling the 'secret' of human longevity is undoubtedly one of the most intriguing challenges for the scientific community. Certainly, genetic factors are amongst the determinants of successful ageing, however, an active lifestyle, especially regular exercise, is also a positive contributor and has been recognized as such for quite some time.

Ageing is a continuum of biological processes characterized by progressive adaptations which can be influenced by both genetic and physiological factors. In terms of human maturation, physically and cognitively functional centenarians certainly represent an impressive example of successful healthy ageing. However, even in these unique individuals, with the passage of time, declining lung function and sarcopenia lead to a progressive fall in maximal strength, maximal oxygen uptake, and therefore reduced exercise capacity. The subsequent mobility limitation can initiate a viscous downward spiral of reduced physical function and health. Emerging literature has shed some light on this multi-factorial decline in function associated with aging and the positive role that exercise and physical capacity can play in the elderly. (Venturelli et al.2012) .

Chronic diseases disproportionately affect older adults and are associated with disability, diminished quality of life, and increased costs for health care and long-term care. Today, about $80 \%$ of older adults have at least one chronic condition, and 50\% have at least two. These conditions can cause years of pain and loss of function.

Regular physical activity seems to be the most important lifestyle behavior that positively influences chronic disease risk factors. For example, a cross-sectional epidemiological study by Ozaki et al. (2003) found that the level of independence, lifestyle, and health of Japanese centenarians (566 men, 1341 women) were significantly correlated with the level of physical activity.

\section{Aims And Objectives}

In this presentation role of lifestyle in healthy ageing was studied. The study was conducted upon 20 subjects aged above 60 years. The anxiety levels of these subjects were assessed by self designed anxiety test.

- The objective was to find out what role did healthy ageing play in the living of the particular age group that is above 60 years of age and their health, happiness, peace and about their contribution to the society.

- The instrument used was a questionnaire comprising questions such as Life achievements, Source of inspiration and Role of life style in healthy ageing. An anxiety test (self-designed) was also given to access the anxiety levels of these subjects

Sample

- The sample consisted of 20 males aged above 60 years. These subjects were people who retired from high posts in the government sector. 


\section{HEALTHY LIFESTYLE}

TABLE

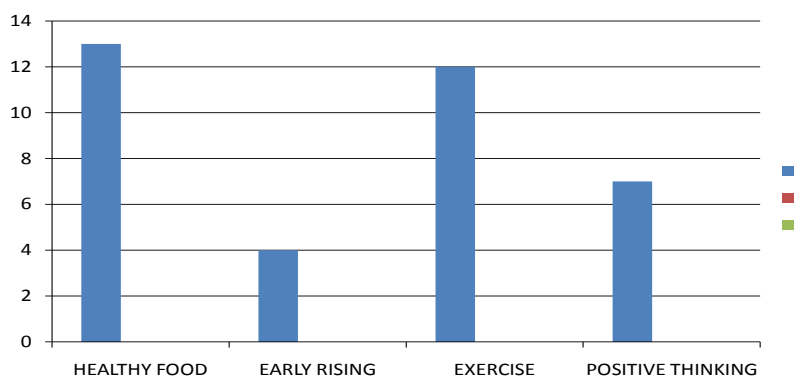

- $4 \%$ contributed their healthy life to early rising.

- $12 \%$ to exercise

- $7 \%$ contributed their health to positive thinking.

- $13 \%$ reported that they ate healthy food.

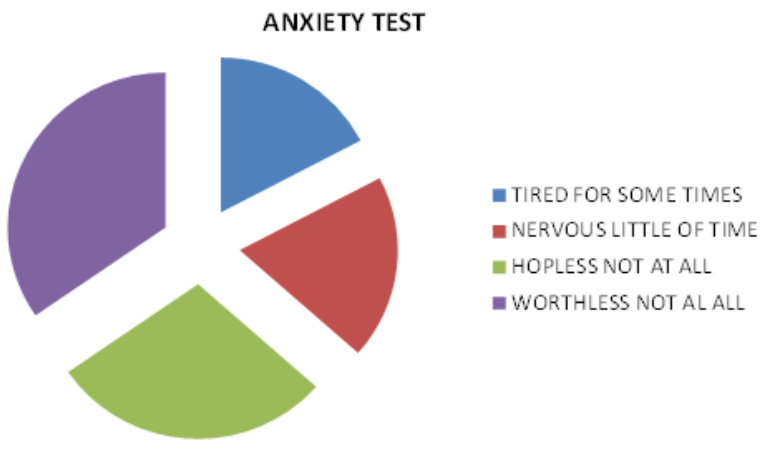

- When asked if they felt tired $45 \%$ said they got tired some times

- $\quad 50 \%$ felt nervous little of the time. Healthy aged people have lesser disease or disability and have high cognitive and physical function and are active.

- $\quad 75 \%$ Did not feel hopeless at all.

- $\quad 90 \%$ Did not feel worthless at all.

\section{Implications Of The Study:}

Promoting good health and healthy behaviours at all ages to prevent or delay the development of chronic disease.

Since non communicable disease in older age is often the consequence of behaviours or exposures earlier in life, strategies need to be put in place that reduce these risks across the life course. Being physically active, eating a healthy diet, avoiding the harmful use of alcohol and not smoking or using tobacco products can all reduce the risk of chronic disease in older age. WHO has identified a set of evidence-based "best buy" interventions for tackling non communicable diseases that are not only highly cost-effective, but also feasible and appropriate to implement within the constraints of low- and middle-income health systems. These include preventive strategies such as taxes on tobacco and alcohol, smoke-free workplaces and public places, reduced salt intake in food and increasing public awareness on diet and physical activity. While health and social care are crucial for older people, numerous determinants of healthy and active ageing lie beyond the health system. Some of these influence older people directly. For example, living in a neighbourhood that is safe, and where other older people can be seen on the streets, might encourage older people to engage more frequently in community activities. But active ageing is a lifelong process, and these same neighbourhoods can also increase the likelihood of younger people being physically active. This is positive for their health and helps to ensure that when they, too, transition to older age, they do so from a stronger base.(WHO 2012)

- They should be persuaded to exercise, encouraged to talk and indulge in social activities, decision making and productive activity, So that they have meaningful lives with lesser anxiety and depression

- Modifiable lifestyle, such as a healthy diet and physical activity, can encourage healthy ageing and improve the quality of life of older people. 


\section{Conclusion}

Population ageing challenges society by increasing demand for acute and primary, health care, straining pension and social security systems and increasing need forlong-term and social care. But older people also make important contributions as family members, volunteers and as active participants in the workforce. They are a significant social and economic resource, and longer life expectancy means a greater opportunity to contribute to society. Where the balance lies between these challengesand opportunities will be determined by how society responds.

Beginning moderately vigorous sports activity, quitting cigarette smoking, maintaining normal blood pressure, and avoiding obesity were separately associated with lower rates of death from all causes and from coronary heart disease among middle aged and older people.

\section{Refrences}

[1] Fratiglioni L, Paillard-Borg S, Winblad B. An active and socially integrated lifestyle in late life might protect against dementia. The Lancet Neurology 2004;3(6):343-353.

[2] Galioto A, Dominguez LJ, Pineo A, et al. Cardiovascular risk factors in centenarians. Experimental Gerontology. 2008;43(February 2):106-13. [PubMed]

[3] Global population ageing: peril or promise? Geneva, World Economic Forum, 2012.

[4] Hillman CH, Erickson KI, Kramer AF. Be smart, exercise your heart: exercise effects on brain and cognition. Nat Rev Neuroscience 2008;9(1):58-65. [PubMed: 18094706].

[5] Menec VH. The Relation Between Everyday Activities and Successful Aging: A 6-Year Longitudinal Study. Journals of Gerontology Series B: Psychological Sciences and Social Sciences 2003;58(2):S74-82.

[6] Ozaki A, Uchiyama M, Tagaya H, Ohida T, Ogihara R. The Japanese Centenarian Study: autonomy was associated with health practices as well as physical status. Journal of the American Geriatrics Society. 2007;55(January 1):95-101. [PubMed]

[7] Perls T, Terry D. Understanding the determinants of exceptional longevity. Annals of Internal Medicine. 2003;139(Septmber 5 Pt 2):445-9. [PubMed]

[8] Venturelli M, Schena F, Scarsini R, Muti E, Richardson RS. Limitations to exercise in female centenarians: evidence that muscular efficiency tempers the impact of failing lungs. Age (Dordrecht) 2012 Jan [PubMed]

[9] World report on disability. Geneva, World Health Organization, 2011. 